\title{
Efficacy of calcium carbonate in reducing the incidence of pre-eclampsia vis-a-vis calcium lactate
}

\author{
Ramani S, Vijaya B, Nithya B \\ Corresponding author: Dr. Vijaya B, MD, Chief Civil Surgeon, Department of obstetrics and \\ gynaecology, Government headquarters hospital, Dindigul, Tamilnadu, India; Email : \\ vijilogudil2@gmail.com
}

Distributed under Attribution-Non Commercial - Share Alike 4.0 International (CC BY-NC-SA 4.0)

\begin{abstract}
Background: Pre-eclampsia remains an important maternal health problem in India. WHO makes a strong recommendation for supplementation of pregnant women with 1.5 grams to 2.0 grams of elemental calcium per day in areas with low dietary calcium intake and for women at high risk of developing hypertensive disorders during pregnancy. Objectives: 1) To evaluate the efficacy of calcium carbonate in reducing the incidence of pre-eclampsia and 2) to study the maternal and fetal outcomes, in antenatal women with hypertensive disorders of pregnancy. Methods: This was a prospective, observational study done in the department of obstetrics and gynaecology, Government Head Quarters Hospital, Dindigul, Tamil Nadu. 600 pregnant women in early second trimester, from 14 weeks to 20 weeks, attending during October 2018 to February 2020, were recruited. The subjects were divided into two groups of 300 each (calcium lactate and calcium carbonate). All the descriptive and inferential statistics were analysed using SPSS software 22 version. P value of $<0.05$ was considered statistically significant. Results: Of 600 antenatal mothers, $86(14.3 \%)$ of them developed preeclampsia in both groups. $8(57 \%)$ mothers of both group, delivered low birth weight babies and $5(36 \%)$ of the babies in calcium lactate group and $3(17 \%)$ calcium carbonate group were admitted in NICU. 45 (100\%) mothers of calcium lactate group had proteinuria and $15(33.3 \%)$ of them had caesarean section. 40 (97.6\%) of calcium carbonate group had proteinuria and $26(63.4 \%)$ of them had caesarean section. Calcium carbonate group showed improved adverse maternal and fetal outcomes. Conclusion: It can be concluded that, calcium supplementation during pregnancy is effective in reducing the risk of pre-eclampsia and adverse maternal and neonatal outcomes associated with the condition.
\end{abstract}

Keywords: Calcium carbonate, calcium lactate, pre-eclampsia, maternal and neonatal outcomes.

Hypertensive disorders of pregnancy (HDP) is the second leading cause maternal mortality affecting approximately $10 \%$ of all pregnancies worldwide ${ }^{1}$. HDP include gestational hypertension, preeclampsia, chronic hypertension and chronic hypertension with superimposed preeclampsia. Amongst these, $10 \%$ to $15 \%$ of direct maternal deaths are associated with preeclampsia and eclampsia ${ }^{2}$. Preeclampsia is a pregnancy specific multi-systemic disorder which is characterized by proteinuria and the onset of hypertension during pregnancy ${ }^{3}$. Eclampsia is the occurrence of otherwise inexplicable convulsions or coma in an already pre-eclamptic patient ${ }^{4}$. Preeclampsia has been believed to be multifactorial in origin, with the presence of both maternal and fetal determinants at risk ${ }^{3}$. Delivery of the placenta is the only known cure for both these conditions. Preeclampsia complicates $7-10 \%$ of pregnancies in the general population ${ }^{5}$. The incidence in developed countries is approximately $3.4 \%$, whereas it varies from $1.8 \%$ to $16.7 \%$ in developing countries ${ }^{6}$.

Preeclampsia and its complications affect both the maternal and foetal outcomes. Maternal risks include severe preeclampsia with end organ damage like renal impairment, HELLP syndrome, acute pulmonary oedema and the lifethreatening eclampsia. Foetal risks include prematurity,

Received: $18^{\text {th }}$ November 2020, Peer review completed: $8^{\text {th }}$ February 2021, Accepted: $7^{\text {th }}$ March 2021.

Ramani S, Vijaya B, Nithya B. Efficacy of calcium carbonate in reducing the incidence of pre-eclampsia vis-a-vis calcium lactate. The New Indian Journal of OBGYN. 2022; 8(2): 278 - 84. 
intrauterine growth restriction and sudden foetal demise due to placental insufficiency ${ }^{7}$. Although outcome is often good with appropriate intervention, preeclampsia can be devastating and life threatening.

Evidence has shown an inverse relationship between high blood pressure and calcium intake ${ }^{8-10}$. Their results have suggested that calcium supplements ( $\geq 1 \mathrm{~g}$ /day) could lower the risk of preeclampsia ${ }^{10}$. As a result, the World Health Organization (WHO) has recommended to supplement calcium for pregnant women especially to high risk population with a low calcium diet. The WHO issued guidelines ${ }^{11}$ recommend routine calcium supplementation for the prevention of preeclampsia. It states that in populations with low calcium intake, calcium supplementation as part of antenatal care particularly among those at higher risk of hypertension. The guideline recommended daily administration of 1.5-2.0 $\mathrm{g}$ of supplemental calcium from 20 weeks of gestation onwards. In India too, the doctors recommend daily dietary allowance (RDA) of $1200 \mathrm{mg}$ per day of calcium during pregnancy and lactation. The National Nutrition Monitoring Bureau (NNMB) - 2012 data from 10 Indian states shows that the daily calcium intake during pregnancy and lactation for Indian women is less than $30 \%$ of RDA (which means it is only $400 \mathrm{mg} / \mathrm{d})^{12}$. This shows that most pregnant and lactating women in India have low dietary calcium intake.

A meta-analysis by Win Khaing ${ }^{13}$ has proved that calcium supplementation may be used for prevention for preeclampsia. Although these pieces of evidence suggest benefits of calcium, yet, it is still unclear which supplement or a combination is most beneficial for preventing preeclampsia and gestational hypertension $(\mathrm{GH})$ or pregnancy induced hypertension (PIH). We therefore conducted this study with the objectives to evaluate the efficacy of calcium carbonate in reducing the incidence of pre-eclampsia, in comparison with calcium lactate, which is being used currently and also to study the maternal and fetal outcomes, in antenatal women with hypertensive disorders of pregnancy.

\section{Materials and methods}

This was a prospective, observational study done in the department of Obstetrics and Gynaecology, Government Head Quarters Hospital, Dindigul, Tamil Nadu. All pregnant women in early second trimester, from 14 weeks to 20 weeks, attending the ANC OPD in the hospital during the period from October 2018 to February 2020, were recruited for inclusion in the study. A total of 600 subjects were included in the study following universal sampling method.
The institutional human ethics committee approved the study. Informed written consent was obtained from all the participants after providing detailed information on objectives of the study. The confidentiality of the study participants was maintained throughout the course.

Inclusion criteria: Participants were parous women whose most recent pregnancy ( $<14$ weeks and above of gestation) had been complicated by pre-eclampsia or eclampsia.

Exclusion criteria: Women were not eligible for the trial if they were younger than 18 years old; were already pregnant; were taking calcium supplementation; had chronic hypertension with persistent proteinuria; had a history or symptoms of urolithiasis, renal disease, or parathyroid disease; ; were using long-term contraception (eg, hormonal injections or implant, intrauterine contraceptive device, or sterilization); or who were under medications (diuretics, digoxin, phenytoin, tetracyclines) and those who were unwilling to give informed consent.

Procedure: 600 pregnant women in early second trimester were recruited for inclusion in the study. After obtaining the consent for participation in the study, 300 pregnant women who had taken calcium lactate tablets from 14 weeks were included in group A (calcium lactate group). 300 pregnant women who had taken calcium carbonate tablets from 14 weeks of gestation were included in group B (calcium carbonate group) twice daily with meals, as per the GOI guidelines 2014. Each calcium lactate tablet 300mg contains $39 \mathrm{mg}$ of elemental calcium and each calcium carbonate tablet contains $500 \mathrm{mg}$ elemental calcium and $250 \mathrm{IU}$ vitamin D3. These women were followed in the antenatal OPD as per the antenatal care protocol of our institute. They were specifically monitored for pre-eclampsia /eclampsia by routine antenatal BP monitoring and test for albuminuria and periodic investigations were done in pre-eclamptic mothers fortnightly to rule out end organ damage like CBC (complete blood count), liver function test (LFT) and renal function test (RFT). In addition, maternal and fetal monitoring was done as per the point of care protocol. Patients were followed till 42 days after delivery for the development of pre-eclampsia and for feto-maternal outcomes.

Outcomes: The incidence of preeclampsia and/or eclampsia was considered as primary maternal outcome. Preeclampsia was defined as an increase in blood pressure $(\geq$ 140/90) associated with a proteinuria or signs of target organ damage occurring after gestational week 20. Hypertension was defined as a blood pressure of $\geq 140 \mathrm{~mm} \mathrm{Hg}$ and/or $\geq 90$ $\mathrm{mm} \mathrm{Hg}$ on 2 occasions at least 6 hours apart. Eclampsia was 
defined as a seizure in a woman with preeclampsia.

Secondary maternal outcomes were systolic and diastolic blood pressures at delivery and at onset of pre-eclampsia, gestational age at delivery, treatment compliance, maternal death/mortality, eclampsia, renal failure, HELLP syndrome, admission to intensive care, placental abruption, caesarean section, severe preeclampsia proteinuria, and woman's hospital stay for 7 days or more.

Fetal outcomes were stillbirth or death before discharge from hospital, death or severe neonatal morbidity, preterm birth (birth before 37 weeks of estimated gestation), neonate small for gestational age, birthweight less than $2500 \mathrm{gm}$, admission to neonatal intensive care unit (NICU) and neonate in intensive care unit for 7 days or more.

Blood pressure measurement and urine analysis: After about five minutes of rest, the systolic and diastolic blood pressures of all participants were measured at each subsequent visit using a standard sphygmomanometer and stethoscope in sitting posture. The first and fifth Korotkoff's phase were recorded as systolic and diastolic blood pressures respectively. Urine samples were collected at enrolment and at each subsequent hospital visit. Midstream urine was collected in a sterile container and analysed in less than an hour with a reactive urine dipstick with colorimetric scale. A qualitative reading of $>2+$ dipstick was considered as positive for proteinuria.

Statistical methods: Preeclampsia, severity of preeclampsia, neonatal outcome and maternal outcomes the primary outcome variable. Study group (group A vs group B) was primary explanatory variable. Descriptive analysis was carried out by mean and standard deviation for quantitative variables, frequency and proportion for categorical variables. Categorical outcomes were compared between study groups using Chi square test. $\mathrm{P}$ value $<0.05$ was considered statistically significant.

\section{Results}

600 people included into final analysis. 86 (14.34\%) people had preeclampsia. Normal blood pressure reported in $255(85 \%)$ antenatal mothers received calcium lactate (group A) and it was 259 (86\%) in calcium carbonate (group B) intake mothers. Preeclampsia was present in $45(15 \%)$ in group $\mathrm{A}$ and $41(14 \%)$ in group $\mathrm{B}$. The difference of proportion for blood pressure between the study groups was statistically not significant (p value 0.64$) .264(88 \%$ ) of the

\begin{tabular}{|c|c|c|c|}
\hline \multirow{2}{*}{$\begin{array}{l}\text { Baseline } \\
\text { parameters }\end{array}$} & \multicolumn{2}{|c|}{ Study groups } & \multirow{2}{*}{$\begin{array}{l}\text { Chi } \\
\text { square test } \\
\text { (p value) }\end{array}$} \\
\hline & $\begin{array}{l}\text { Group A } \\
(\mathbf{N}=\mathbf{3 0 0})\end{array}$ & $\begin{array}{l}\text { Group B } \\
(\mathbf{N}=\mathbf{3 0 0})\end{array}$ & \\
\hline \multicolumn{4}{|l|}{ Blood pressure } \\
\hline Normal blood pressure & $255(85 \%)$ & $259(86 \%)$ & \multirow[t]{2}{*}{0.64} \\
\hline Preeclampsia & $45(15 \%)$ & $41(14 \%)$ & \\
\hline \multicolumn{4}{|l|}{ Age } \\
\hline$<20 \mathrm{yrs}$ & $31(10.3 \%)$ & $25(8.3 \%)$ & \multirow[t]{3}{*}{0.53} \\
\hline $20-35$ yrs & $264(88 \%)$ & $272(90.7 \%)$ & \\
\hline$>35 \mathrm{yrs}$ & $5(1.7 \%)$ & $3(1 \%)$ & \\
\hline \multicolumn{4}{|l|}{ BMI category } \\
\hline Underweight (BMI $<18.5 \mathrm{~kg} / \mathrm{m}^{2}$ ) & $2(0.7 \%)$ & $20(6.6 \%)$ & \multirow[t]{4}{*}{0.001} \\
\hline Normal weight (BMI18.5-22.9 kg/m²) & $46(15.3 \%)$ & $90(30 \%)$ & \\
\hline Normal weight (BMI18.5-22.9 kg/m²) & $39(13 \%)$ & $38(12.7 \%)$ & \\
\hline Obese $\left(\right.$ BMI $\left.>25 \mathrm{~kg} / \mathrm{m}^{2}\right)$ & $213(71 \%)$ & $152(50.7 \%)$ & \\
\hline \multicolumn{4}{|l|}{ Parity } \\
\hline Primigravida & $146(48.7 \%)$ & $121(40.3 \%)$ & \multirow[t]{2}{*}{0.04} \\
\hline Multigravida & $154(51.3 \%)$ & $179(59.7 \%)$ & \\
\hline
\end{tabular}

antenatal mothers who received calcium lactate and $272(90.7 \%)$ who received calcium carbonate were aged between 20-30 years. The difference of proportion for age group between the study groups was statistically not significant ( $\mathrm{p}$ value 0.53 ). 213(71\%) of antenatal mothers who received calcium lactate and $152(50.7 \%)$ of those who received calcium carbonate were obese. The difference of proportion for BMI between the study groups was statistically significant ( $p$ value 0.001 ). 154(51.3\%) of antenatal mothers who received calcium lactate and $179(59.7 \%)$ of those who received calcium carbonate were multigravida. The difference of proportion for parity between the study groups was statistically significant ( $p$ value 0.04 ) (table 1).

Severe preeclampsia was reported in 14(31\%) of antenatal mothers who received calcium lactate and it was $16(39 \%)$ in mothers who took calcium carbonate. Non severe preeclampsia was present in $31(69 \%)$ in group A and $25(61 \%)$ in group B. The difference proportion for severity blood pressure between the study groups was statistically not significant ( $p$ value 0.44 ). The women who took calcium lactate $33(73 \%)$ of them were aged between 20 to 35 years and $8(17 \%)$ of them were aged $<20$ years and the women who took calcium carbonate $33(80 \%)$ of them were between 20 to 35 years of age group and $6(15 \%)$ of them were aged $<20$ years. The difference of proportion for age group among preeclampsia mother between the study group was statistically not significant ( $\mathrm{p}$ value 0.681 ). The women who took calcium lactate $36(80 \%)$ of them were obese and $5(11 \%)$ of them had normal BMI and the women who took calcium carbonate $30(74 \%)$ of them were obese and $5(12 \%)$ of them had normal BMI. The women who took calcium lactate $18(40 \%)$ of them were multigravida and $27(60 \%)$ of them were primigravida and the women who took calcium 
carbonate $22(54 \%)$ of them were multigravida and $19(46 \%)$ of them were primigravida. The difference of proportion for parity among preeclampsia mother between the study group was statistically not significant ( $\mathrm{p}$ value 0.20 ).

The women who took calcium lactate all $45(100 \%)$ of them had proteinuria and $15(33.3 \%)$ of them undergone caesarean section. The women who took calcium carbonate $40(97.6 \%)$ of them had proteinuria and $26(63.4 \%)$ of them developed and developing countries and their pooled estimate had shown that calcium supplementation during pregnancy significantly reduced occurrence of gestational hypertension [RR $0.70,95 \%$ CI $0.57-0.86]$ and preeclampsia [RR $0.48,95 \%$ CI $0.33-0.69]$ as well as a reduction in the risk of prematurity [RR $0.81,95 \%$ CI $0.64-$ 1.03].

In our study, out of 600 antenatal mothers, 86 (14.3\%) developed preeclampsia. This is similar to other studies ${ }^{14-16}$ in which the incidence was found to vary from $1.8 \%$ to $16.7 \%$ in developing countries. According to NFHS-4, the incidence of preeclampsia in India was found to be $52 \%{ }^{17}$.

So, what could be the explanation of protective effect of calcium supplementation during pregnancy? Low calcium intake has been hypothesized to cause increase in blood pressure by stimulating the release of parathyroid hormone and/or renin leading to an increase in intracellular calcium concentration in vascular smooth muscle cells, thereby causing vasoconstriction. Role of calcium supplementation in reducing hypertensive disorders in pregnancy can possibly be explained by reduction in parathyroid calcium release and intracellular calcium concentration, thereby reducing smooth muscle contractility and promoting vasodilatation ${ }^{5}$. Calcium supplementation could also prevent preterm labor and delivery by reducing uterine smooth muscle contractility directly and indirectly by increasing magnesium levels ${ }^{18}$.

In antenatal mothers who had taken calcium lactate tablets, $18 \%$ developed preeclampsia. In calcium carbonate consumed mothers, the incidence was $15.8 \%$ which was lower when compared to the calcium lactate group; but the difference in the incidence was not statistically significant. In group A, 68.9\% developed nonsevere preeclampsia and $31.1 \%$ had severe preeclampsia. In group B, $61 \%$ had nonsevere preeclampsia and $39 \%$ had severe preeclampsia. Since no study comparing the efficacy of calcium carbonate with lactate in reducing the incidence of preeclampsia, was available, those studies that compared the calcium intake and placebo were included for comparison. Cochrane review 2018 (51) by Hofmeyer et al ${ }^{20}$, compared the high dose $(>1 \mathrm{~g} /$ day $)$ and low dose $(<1 \mathrm{~g} /$ day $)$ calcium supplementation with placebo and concluded that high dose calcium supplementation reduced the risk of preeclampsia. 
Studies have shown that advanced maternal age is an independent risk factor for development of preeclampsia ${ }^{20}$. The risk of developing preeclampsia is highest amongst women $<20$ years of age, but women $\geq 35$ years of age also have an increased risk of developing preeclampsia ${ }^{20}$. In our study, only $7 \%$ antenatal mothers above 35 yrs developed preeclampsia. This might be because of the reason that majority of the study population belonged to the age group $20-35 \mathrm{yrs}$, as early marriage was more common in our study area. In calcium lactate group, $17.8 \%$ were below 20 yrs, $73.3 \%$ were between $20-35$ years and $8.9 \%$ were above 35 yrs. Whereas in calcium carbonate group, $14.6 \%$ were below 20 yrs, $80.5 \%$ were between $20-35$ years and $2(4.9 \%)$ were above 35 yrs. Nulliparity is the most known risk factor for preeclampsia. Nulliparous women are three times more at risk of developing preeclampsia than multiparous women ${ }^{21}$. In our study, $53 \%$ were nulliparous. In calcium lactate group, $60 \%$ were primigravida and $40 \%$ were multigravida. In calcium carbonate group, $48.7 \%$ were primigravida and $51.3 \%$ were multigravida. This is in accordance with studies by Sajith et al ${ }^{15}(53.8 \%)$, Cherian et $\mathrm{al}^{20}(61.2 \%)$ and Agrawal et $\mathrm{al}^{22}(31.5 \%)$.

The connection between obesity and preeclampsia has been linked to inflammation. Obesity is often linked with hyperglycaemia, causing haemoglobin to pick up glucose and lose its affinity for oxygen. This leads to placental hypoxia, leading to an inflammatory response, the release of cytokines, and subsequent endothelial dysfunction of preeclampsia. WHO estimates that the female prevalence of overweight and obesity is $18 \%$ in India ${ }^{23}$. This connection was also observed in our study where $76.7 \%$ of the preeclamptic mothers had a BMI $>25 \mathrm{~kg} / \mathrm{m} 2$. In calcium lactate group, $80 \%$ were obese. In calcium carbonate group, $73.2 \%$ were obese. The incidence of preeclampsia in obese mothers, in calcium carbonate group was found to be little less when compared to the lactate group; but difference is not statistically significant. Hence, we advise weight control before conception in case of overweight mothers, as obesity not only raises the risk of preeclampsia, but other diseases also.

Maternal outcomes were observed in normotensive mothers as well as in preeclamptic mothers. The complications were more in the preeclamptic group. However, in our study, we didn't encounter maternal mortality, also seen in the study by Praveen at al ${ }^{20}$. Our preeclamptic mothers didn't develop eclampsia, HELLP syndrome, pulmonary edema, renal failure. This may be due to their adherence to regular antenatal check-up, follow up by the VHNs by home visit and early identification of complications. In our study, the caesarean section rate was $47.7 \%$ in preeclamptic mothers, which was higher when compared to $33.7 \%$ in normotensive mothers. In calcium lactate group, $33.3 \%$ delivered by caesarean section. In calcium carbonate group, $63.4 \%$ delivered by caesarean section, which was more than in the lactate group. In our study, hospital stay was more in preeclamptic mothers. $34.9 \%$ had a prolonged hospital stay of more than 7 days. None had ICU admission. In calcium lactate group, $20 \%$ had prolonged hospital stay of $>7$ days. In calcium carbonate group, $34.9 \%$ had prolonged hospital stay of $>7$ days, which was more when compared to the calcium lactate group.

Neonatal outcomes were also analysed in our study. Out of the 86 preeclamptic deliveries, $2.3 \%$ had intrauterine death, $7 \%$ had preterm deliveries, $18.6 \%$ had low birth weight babies out of which $9.3 \%$ had NICU admission. All these complications were more when compared to the normotensive group. Group A had no intrauterine death, $2.2 \%$ had preterm deliveries, $17.8 \%$ had low birth weight babies out of which $11.1 \%$ had NICU admission. Whereas, in group B, $4.9 \%$ had intrauterine death, $12.2 \%$ had preterm deliveries, $19.5 \%$ had low birth weight babies out of which $7.3 \%$ had NICU admission. Similar findings were seen in a study done by Parveen et al ${ }^{20}$ found that the most common neonatal complication was prematurity $(23.65 \%)$, low birth weight $(7.52 \%)$ and intra uterine growth restriction $(9.67 \%)$. The perinatal mortality constituted about $15 \%$, which included intra uterine demise of the fetus (8.6\%), still births $(2.15 \%)$ and neonatal deaths (4.3\%).

Limitations and recommendations: Few limitations of this study include: a) a small size; b) compliance of the mothers towards calcium tablet intake was not sure; in spite of adequate counselling given to them during their antenatal visits. Long term studies need to be conducted on this topic so as to validate the results in our study.

Pre-eclampsia should be identified as a priority area in improving maternal health and thus prevention, early detection and timely management of pre-eclampsia and its risk factors at antenatal care visits is important in order to bring about considerable improvement in maternal and perinatal health in India. Hence, we advise all the pregnant mothers to strictly be compliant towards the consumption of calcium carbonate with vitamin D tablets as provided by Govt. of India. Proper education regarding anaemia prophylaxis and treatment reduces the anaemia incidence, thereby reducing the risk of preeclampsia. Pregnant mothers have to be taught about the timing of calcium and IFA tablets 
intake, as well as to recognise the imminent signs and symptoms of severe preeclampsia before the intrauterine demise of foetus occurs or mother develops one of the grave complications. Also, we advise reduction of weight before conception, as obesity is a modifiable risk factor for preeclampsia.

\section{Conclusion}

The findings of this study strongly show a positive correlation between calcium intake during pregnancy reduces preeclampsia. We recommend routine calcium carbonate supplementation as per GOI guidelines in all antenatal women from early pregnancy, which might reduce the severity of maternal and fetal outcome in hypertensive pregnancies.

Acknowledgements: We acknowledge the technical support in data entry, analysis and manuscript editing by "Evidencian Research Associates".

\section{Conflict of interest: None. Disclaimer: Nil.}

\section{References}

1. Cong KJ. Calcium and pregnancy induced hypertension. Zhonghua Fu Chan Ke Za Zhi. 1993; 28(11): 657-9.

2. An L, Li W, Xie T, Peng X, Li B, Xie S, et al. Calcium supplementation reducing the risk of hypertensive disorders of pregnancy and related problems: A meta-analysis of multicentre randomized controlled trials. Int J Nurs Pract. 2015; 21:19-31.

3. Cudihy D, Lee RV. The pathophysiology of preeclampsia: current clinical concepts. J Obstet Gynaecol (Lahore). 2009; 29(7): 576-82.

4. Cheng MH, Wang PH. Placentation abnormalities in the pathophysiology of preeclampsia. Expert Rev Mol Diagn. 2009; 9(1): 37-49.

5. Aghamohammadi A, Zafari M. Calcium supplementation in pregnancy and prevention of hypertensive disorders in elderly women. Sci Asia. 2015; 41: 259-62.

6. Khaing W, Vallibhakara SAO, Tantrakul V, Vallibhakara O, Rattanasiri S, McEvoy $M$, et al. Calcium and vitamin D supplementation for prevention of preeclampsia: a systematic review and network metaanalysis. Nutrients. 2017; 9(10):1141.

7. Uzan J, Carbonnel M, Piconne O, Asmar R, Ayoubi JM. Pre-eclampsia: pathophysiology, diagnosis, and management. Vasc Health Risk Manag. 2011; 7: 467.
8. Villar J, Belizan JM, Fischer PJ. Epidemiologic observations on the relationship between calcium intake and eclampsia. Int J Gynecol Obstet. 1983; 21(4): 271-8.

9. Hofmeyr GJ, Atallah AN, Duley L. Calcium supplementation during pregnancy for preventing hypertensive disorders and related problems (Cochrane Review). Cochrane Libr. 2006; 3: CD001059.

10. Hofmeyr GJ, Lawrie TA, Atallah ÁN, Torloni MR. Calcium supplementation during pregnancy for preventing hypertensive disorders and related problems. Cochrane database Syst Rev. 2018; 10(10): CD001059.

11. Duley L. The global impact of pre-eclampsia and eclampsia. Semin Perinatol. 2009; 33(3):130-7

12. Thomas M, Weisman SM. Calcium supplementation during pregnancy and lactation: effects on the mother and the fetus. Am J Obstet Gynecol. 2006;194(4): 937-45.

13. Khaing W, Arj-Ong Vallibhakara S. Calcium and Vitamin D Supplementation for Prevention of Preeclampsia: A Systematic Review and Network MetaAnalysis Nutrients. 2017; 9: 1141

14. Magee LA, Sharma S, Nathan HL, Adetoro OO, Bellad $\mathrm{MB}$, Goudar S, et al. The incidence of pregnancy hypertension in India, Pakistan, Mozambique, and Nigeria: A prospective population-level analysis. PLoS Med. 2019;16(4): e1002783.

15. Sajith M, Nimbargi V, Modi A, Sumariya R, Pawar A. Incidence of pregnancy induced hypertension and prescription pattern of antihypertensive drugs in pregnancy. Int J Pharma Sci Res. 2014; 23: 4.

16. Andarge RB, Anshebo AA, Halil HM, Kebede BA, Abdo RA. Prevalence and Associated Factors of Preeclampsia among Pregnant Women at Antenatal Booking in the Halaba Kullito General Hospital, Southern Ethiopia. J Women's Heal Care. 2020; 9(496): 420-2167.

17. Choorakuttil RM, Devarajan P, Rajalingam B, Jain N, Sharma LK, Nagar S, et al. Samrakshan: Rationale for universal 1 st trimester screening to identify pregnant women at risk for preterm preeclampsia. Journal of fetal radiology. 2020. Accesed from: http://fetalradiology.in /2020/03/20/samrakshan-1st-trimester-screeningidentifies-1-in-5-pregnant-women-as-high-risk-forpreterm-preeclampsia-and-fetal-growth-restriction/

18. Carroli G, Merialdi M, Wojdyla D, Abalos E, Campodonico L, Yao S-E, et al. Effects of calcium supplementation on uteroplacental and fetoplacental blood flow in low-calcium-intake mothers: a 
The New Indian Journal of OBGYN. 2021 (January-June);8(2)

randomized controlled trial. Am J Obstet Gynecol. 2010; 202(1): 45-e1.

19. Hofmeyr GJ, Duley L, Atallah A. Dietary calcium supplementation for prevention of pre-eclampsia and related problems: a systematic review and commentary. BJOG An Int J Obstet Gynaecol. 2007;114(8):933-43.

20. Aabidha PM, Cherian AG, Paul E, Helan J. Maternal and fetal outcome in pre-eclampsia in a secondary care hospital in South India. J Fam Med Prim care. 2015; 4(2):257.

21. Duckitt K, Harrington D. Risk factors for pre-eclampsia at antenatal booking: systematic review of controlled studies. Bmj. 2005; 330(7491): 565.

22. Agrawal S, Walia GK. Prevalence and risk factors for symptoms suggestive of preeclampsia in Indian women. J Women's Heal. 2014; 3(6): 2-9.
23. Lopez-Jaramillo P, Barajas J, Rueda-Quijano SM, Lopez-Lopez C, Felix C. Obesity and preeclampsia: common pathophysiological mechanisms. Front Physiol. 2018; 9:1838.

\section{Ramani $\mathrm{S}^{1}$, Vijaya $\mathrm{B}^{2}$, Nithya $\mathrm{B}^{3}$}

${ }^{1}$ Post graduate, Department of obstetrics and gynaecology, Government headquarters hospital, Dindigul, Tamilnadu, India; ${ }^{2}$ Chief Civil Surgeon, Department of obstetrics and gynaecology, Government headquarters hospital, Dindigul, Tamilnadu, India; ${ }^{3}$ Associate professor, Department of biochemistry, HKRK college, Theni, Tamilnadu, India. 